\title{
Besitzstand, Fähigkeitsausweis und die Spartendatenbank
}

Christian Bernath

Korrespondenz:

Dr. med. Christian Bernath

Co-Präsident SGDP

Ludretikonerstrasse 53

CH-8800 Thalwil

Tel. 0447211093

cbernath@hin.ch

\section{Besitzstandswahrung}

Die delegierte Psychotherapie ist als Besitzstand anders geregelt als die übrigen Dignitäten.

Wer vor der Einführung des TARMED delegierte Psychotherapie ausgeübt hat, aber über keinen Facharzttitel für Psychiatrie und Psychotherapie oder Kinder- und Jugendpsychiatrie und -psychotherapie verfügt, muss den Fähigkeitsausweis «Delegierte Psychotherapie FMPP» beantragen und anschliessend die dort definierte Fortbildungspflicht erfüllen.

Nur wer neu mit delegierter Psychotherapie beginnen will und nicht Psychiaterin oder Psychiater ist, muss das entsprechende Curriculum von 60 Stunden zu definierten Inhalten absolvieren, um den Fähigkeitsausweis Delegierte Psychotherapie zu erlangen.

Die Unterlagen zur Erlangung des Fähigkeitsausweises finden Sie auf der Homepage der Schweizerischen Ärztegesellschaft für Delegierte Psychotherapie (SGDP): www.sgdp.ch.

\section{Spartendatenbank}

Die santésuisse hat alle ihre angeschlossenen Kassen darüber informiert, dass nur noch den Ärztinnen und Ärzten delegierte Psychotherapie vergütet wird, die in der Spartendatenbank von TARMED Suisse aufgeführt sind. Andere Listen sind nicht mehr gültig.

Es haben sich aber noch nicht alle delegierenden Kolleginnen und Kollegen bei der Spartendatenbank angemeldet, weil ihnen der Unterschied zwischen der Dignität und Sparte nicht ganz klar geworden ist.

In der Dignitätsdatenbank geht es um die persönlichen Qualifikationen der Ärztin und des Arztes. Psychiaterinnen und Psychiater mit Facharzttitel haben a priori auch die Dignität «Delegierte Psychotherapie» und müssen dies nicht speziell deklarieren.

Bei der Spartendatenbank geht es hingegen um das ganze Leistungspaket der delegierten Psychotherapie und umfasst damit auch die Qualifikation der Therapeutinnen und Therapeuten, die
Anstellungsbedingungen und die Arbeitsverhältnisse. Mit der Meldung an die Spartendatenbank bezeuge ich, dass ich und meine Psychotherapeutinnen und Psychotherapeuten die qualitativen und rechtlichen Bedingungen der delegierten Psychotherapie erfüllen.

Das heisst, auch Psychiaterinnen und Psychiater mit Facharzttitel müssen sich bei der Spartendatenbank melden, wenn sie delegierte Psychotherapie ausüben.

Das entsprechende Meldeformular finden Sie unter www.sgdp.ch $\rightarrow$ Aktualitäten $\rightarrow$ Spartendatenbank oder unter www.fmh.ch $\rightarrow$ Unsere Dienstleistungen $\rightarrow$ Tarife $\rightarrow$ TARMED $\rightarrow$ Delegierte Psychotherapie.

Wer den Fähigkeitsausweis «Delegierte Psychotherapie FMPP» erwirbt, wird auf die Spartendatenbank gesetzt und muss nicht noch speziell Meldung erstatten.

Bitte beachten Sie, dass bei den Psychotherapeuten immer noch die alte Formulierung verwendet wird, die im neuen Jahr noch bearbeitet wird. Alle Psychotherapeuten fallen deshalb noch unter die Übergangsregelungen, die im Gegensatz zu den Ärztinnen und Ärzten nicht dem Dignitätskonzept unterliegen (Dauer der Übergangszeit: 3 Jahre), sondern in Anlehnung an die zeitliche Dauer des Freizügigkeitsgesetzes mindestens 7 Jahre (Beilage G der Vereinbarung über die Anerkennung von Sparten nach TARMED). Die TARMED Suisse hat die Krankenkassen im Januar 2007 entsprechend informiert und es sollten keine Beanstandungen mehr erfolgen. Das bedeutet, dass alle Psychotherapeutinnen und Psychotherapeuten, die schon vor der Einführung des TARMED während dreier Jahre delegiert gearbeitet haben, ohne weiteres weiterarbeiten können.

Wer über eine Praxisbewilligung für die selbständige psychotherapeutische Tätigkeit verfügt, ist ebenfalls berechtigt, delegiert zu arbeiten. Hier gilt der Grundsatz, dass eine gesetzliche Regelung (auch auf kantonaler Ebene) Vorrang hat gegenüber einem Tarifvertrag. 